\title{
LD Bodies From Blood Buffy Coat: an Easy Approach for Definitive Diagnosis of Visceral Leishmaniasis
}

\author{
AKM Shamsuzzaman, ${ }^{1}$ Md. Chand Mahmud, ${ }^{1}$ Shamima Akhter, ${ }^{2}$ AKM Musa, ${ }^{1}$ Md. Akram Hossain ${ }^{1}$ \\ ${ }^{1}$ Department of Microbiology, Mymensingh Medical College, Mymensingh, 2Department of Biochemistry, Shaheed Ziaur Rahman \\ Medical College, Bogra
}

\begin{abstract}
This observational study was carried out as a preliminary one to see the effectiveness and reliability of an easy noninvasive approach for the confirmatory diagnosis of Visceral Leishmaniasis (VL) by detection of amastigote forms (LD bodies) of the parasite. Clinically suspected 73 febrile cases were investigated by examination of bone marrow/ splenic aspirate to detect LD bodies, of whom 57 (41.61\%) showed LD positive result on microscopy and/or culture in modified Novy, Nichole, MacNeal (NNN) medium. Blood buffy coat was obtained by the method of density gradient centrifugation using mono-nuclear cell separation fluid "Lymphoprep" from all the cases confirmed as VL. Thin smears on microscopic slides were prepared from all 57 buffy coats, and stained by Leishman stain to see LD bodies microscopically. Out of 57 buffy coat smears, 53 (92.98\%) yielded LD bodies. Findings of the present study has encouraged to carry out a field trial so that this easy approach can be practiced as a clinical diagnostic procedure.
\end{abstract}

Key words: Diagnosis of Visceral Leishmaniasis, LD body, Buffy coat

\section{Introduction}

Definitive diagnosis of Visceral Leishmaniasis (VL) is based on the demonstration of amastigote form (LD body) of Leishmania donovani in tissue specimens like splenic aspirates, bone marrow or peripheral blood. ${ }^{1}$ Microscopy of bone marrow aspirates is highly sensitive diagnostic approach for paediatric patients, because of higher parasitization. In adult patients, sensitivity of bone marrow microscopy for LD body is around $70 \% .^{2}$ But, the procedure of marrow aspiration either from illiac crest or from sternum is very much painful. Microscopic examination of splenic aspirates offers the highest sensitivity (up to 98\%), but is associated with the risk of life-threatening haemorrhage in cases with profound thrombocytopenia. ${ }^{3}$ Culture on Novy, Nichole, MacNeal (NNN) or Schneider's media have been found to yield successful results. ${ }^{4}$

Correspondence:

Dr. AKM Shamsuzzaman, Assistant Professor, Department of Microbiology, Mymensingh Medical College, Mymensingh

E-mail: stusar_mmc@yahoo.com
Serological diagnosis is based on the presence of specific humoral antibodies in cases of VL. There are a range of serological methods available for the diagnosis of VL with varying sensitivity and specificity. The Indirect Fluorescent Antibody Test (IFAT) is one of the most sensitive tests available. The test is based on detecting antibodies which are demonstrated in the very early stages of infection and remain undetectable during 6-9 months after cure. If the antibodies persist in low titres, it is a good indication of a probable relapse. Titres above $1 / 20$ are significant and above $1 / 120$ are diagnostic for IFAT.2,5,6 The Direct Agglutination Test (DAT) is a highly specific and sensitive test. It is a cheaper test and simpler to perform making it an ideal for both field and laboratory use. The antigen is prepared from promastigotes of L. donovani and test can be carried out on plasma, serum, blood spots collected on filter paper and whole blood. Serum titres of 1:32,000, in case of DAT, are considered positive. ${ }^{7}$ The Enzyme Linked Immunosorbent Assay (ELISA) is 100\% specific with a sensitivity above $98 \%$. The antigen is prepared from promastigotes of $L$. donovani and the test can be performed on serum, plasma or blood spots collected on filter paper. It is useful in the field owing to its simplicity. 
However, false positive results are not uncommon. Hence, it is preferred less than the IFAT and DAT for laboratory diagnosis. ${ }^{8}$

Another interesting field for definitive diagnosis of KalaAzar (KA) might be the detection of LD body from buffy coat smear of peripheral blood. Because, when blood is settled in presence of an anticoagulant, the WBCs are deposited and concentrated in buffy coat at the interface of $\mathrm{RBCs}$ and plasma. If buffy coat is considered for making a smear, it is supposed to show high content of WBCs including monocytes. The monocytes parasitized by LD bodies, if present, will be higher in the smear. Thus the rate of LD body positivity is expected to be higher. ${ }^{9}$

In Bangladesh, bone marrow or splenic aspirates are examined conventionally for LD body detection. ${ }^{10}$ Undoubtedly, collection of either of the specimens is troublesome and inconvenient for both patients and doctors due to the excruciating pain and procedural complications. As a result, patients remain reluctant to be investigated. On the other hand, buffy coat is obtained from peripheral blood and collection of the specimen is easier and widely acceptable to the patients. ${ }^{11}$ No investigators from Bangladesh has yet reported or even known to have studied on LD body detection from buffy coat. Unquestionably, buffy coat preparation and examination is easier than splenic aspirate collection, preparation and examination.

\section{Methods}

This observational study was done in the Department of Microbiology of Mymensingh Medical College (MMC) during the period from November, 2003 to March, 2004. Patients were selected from the inpatient departments of MMC Hospital, Community Based Medical College Hospital and as community cases from nearby subdistricts of Fulbaria and Trishal. A total of 71 patients were investigated for diagnosis of Kala-azar on the basis of prolonged low grade irregular fever with spleno-hepatomegaly, emaciation, anaemia and other related clinical findings.

All patients underwent preliminary testing for routine haematological profile, e.g., $\mathrm{Hb} \%$, ESR, Total Leukocyte Count (TLC), Differential Leukocyte Count (DLC), Total Platelet count (TPC) and Peripheral Blood Film (PBF). Serum samples were tested for Aldehyde test (AT) and antiK39 antibodies by Immunochromatographic Test (ICT) strips (Inbios Inc., USA). From all the patients, aspirates from bone marrow/ spleen were collected and examined by microscopy for LD bodies and by culture for promastigote form of Leishmania donovani.

Bone marrow was aspirated from anterior iliac crest using bone marrow needle, whereas splenic aspirate was taken by syringing according to standard method.12 An appropriate aliquot (1-2 drops) of the specimens was immediately inoculated into the water of condensation of modified NNN media with all aseptic precautions and rest was used to prepare smear for microscopic examination using Leishman stain. For grading and reporting of LD bodies by microscopy, a WHO manual was followed. ${ }^{13}$ After inoculation, culture tubes were incubated at $22-24^{\circ} \mathrm{C}$ and checked daily from 3rd day onwards up to 2 weeks for growth of promastigotes. For the purpose of observing the promastigotes, one drop of the fluid was gently aspirated from liquid phase of the medium by sterile disposable syringe, taking all aseptic precautions and was covered by a cover slip to see motile promastigotes under high power objectives. If no promastigote was found within 2 weeks, the specimen was then considered as negative for culture.

Patients positive for L. donovani in microscopy and/or culture were selected for buffy coat examination. For this purpose, 2 $\mathrm{ml}$. venous blood was collected in a sterile centrifuge tube containing appropriate amount of EDTA. Buffy coat was obtained by centrifuging the tube in an oblique bored centrifuge machine at $3000 \mathrm{rpm}$ for 15 minutes applying the principle of "Density Gradient Centrifugation". Lymphoprep solution was used for that purpose at a ratio of 1:1. A thick and opaque buffy coat $(>1 \mathrm{~mm})$ was formed at the interface of lymphoprep and plasma. The RBCs were settled at the bottom of the tube. ${ }^{14}$ Material from buffy coat was gently sucked by a Pasteur pipette and a thin smear was prepared on microscopic slides. The smears were stained by Leishman stain for detection of LD bodies. Data was recorded in a formatted data sheet and analyzed manually using appropriate statistical formulae.

\section{Results}

In this study, out of 71 suspected cases of prolonged fever, 57 were diagnosed as parasite positive cases of Kala-azar. Majority of the cases were in the age range between 2-17 years. Male to female ratio was 1.38:1. (Table I)

Results of microscopy observing LD bodies showed that bone marrow smears were positive in $(52 / 71,78.78 \%)$ specimens, and all of the tested smears of splenic aspirate $(05 / 05,100 \%)$ were positive. Culture in NNN media showed promastigotes 
among 50/71 (70.42\%) suspected cases. So, microscopy and culture methods together identified 57/71 (80.28\%) positive cases of Kala-azar. The ICT (anti-k39 antibodies) were positive in all $(57 / 57,100.0 \%)$ of the positive cases of Kala-azar. Buffy coat smears for LD bodies were examined among all positive cases and some 53 (92.98\%) were found positive. (Table II)

Table I: Age and sex distribution of confirmed Kala-azar cases

\begin{tabular}{lll}
\hline $\begin{array}{l}\text { Age group } \\
\text { in years }\end{array}$ & Male & Female \\
\hline $2-7(\mathrm{n}=13)$ & $09(69.23 \%)$ & $04(30.77 \%)$ \\
$>7-12(\mathrm{n}=17)$ & $10(58.82 \%)$ & $07(41.18 \%)$ \\
$>12-17(\mathrm{n}=09)$ & $04(44.44 \%)$ & $05(55.56 \%)$ \\
$>17-22(\mathrm{n}=06)$ & $04(66.67 \%)$ & $02(33.33 \%)$ \\
$>22-27(\mathrm{n}=03)$ & $03(100.0 \%)$ & $00(00 \%)$ \\
$>27-32(\mathrm{n}=09)$ & $03(33.33 \%)$ & $06(66.67 \%)$ \\
Total $(\mathrm{n}=57)$ & $33(57.89 \%)$ & $24(42.11 \%)$ \\
\hline
\end{tabular}

Table II: Findings of bone marrow/ splenic aspirates, anti-k39 ICT and LD bodies in buffy coat smears

\begin{tabular}{lll}
\hline Name of the test & $\begin{array}{l}\text { Done in } \\
\text { cases }\end{array}$ & $\begin{array}{l}\text { No (\%) of } \\
\text { positive cases }\end{array}$ \\
\hline $\begin{array}{l}\text { Bone marrow for LD } \\
\text { bodies in microscopy }\end{array}$ & 66 & $52(78.78 \%)$ \\
$\begin{array}{l}\text { Splenic aspirates for LD } \\
\text { bodies in microscopy }\end{array}$ & 05 & $05(100 \%)$ \\
$\begin{array}{l}\text { Culture of bone marrow } \\
\text { and splenic aspirates in }\end{array}$ & 71 & $50(70.42 \%)$ \\
$\begin{array}{l}\text { NNN medium } \\
\text { ICT (anti-k 39 } \\
\text { antibodies) }\end{array}$ & 71 & $57(80.28 \%)$ \\
$\begin{array}{l}\text { Buffy coat smear for LD } \\
\text { bodies }\end{array}$ & 57 & $53(92.94 \%)$ \\
\hline
\end{tabular}

Each of the Kala-azar cases showed a high ESR, reduced haemoglobin concentrations, reduced TLC, increased percentages of lymphocytes in DLC and reduced TPC. (Table III)

Table III: Haematological findings of 57 confirmed cases of Kala-azar

\begin{tabular}{ll}
\hline Name of test & Mean \pm Standard deviation \\
\hline ESR in mm 1st hour & $96.25 \pm 18.76$ \\
Haemoglobin concentration (\%) & $45.35 \pm 6.07$ \\
TLC in thousands & $3678.95 \pm 1463.82$ \\
$\%$ of lymphocytes & $67.25 \pm 4.99$ \\
TPC in thousands & $124.03 \pm 42.59$ \\
\hline
\end{tabular}

\section{Discussion}

The present study explored the reliability and effectiveness of LD body detection in blood buffy coat smear for definitive diagnosis of Kala-azar. The criteria followed to select Kalaazar cases in the present study was in agreement with other reports. ${ }^{15,16}$

Majority of confirmed cases in the present study were in the age range between 2-17 years. Ali and Ashford (1994) found that majority of the cases $(58.0 \%)$ were children below 15 years out of 142 cases of VL. ${ }^{17}$ Benzamin et al (1984) observed that majority patients of VL were in the age group of 5-15 years. ${ }^{18}$ Higher incidence of KA in children might be due to the reason that children of poor family generally suffer from malnutrition. Consequently their immunity is hampered increasing the risk of getting KA infection and developing severe disease. ${ }^{19}$

In the present study, male to female ratio is $1.38: 1$. Another study from home also reported almost similar ratio between male to female. ${ }^{20}$ Males are infected more often than females, most likely because of their increased exposure to sand flies due to professional activities. ${ }^{21}$

Positive results in the present study to see LD bodies by microscopy from different specimens were as follows: bone marrow $78.78 \%$, splenic aspirates $100 \%$, buffy coat smear $92.94 \%$. Result of culture-positivity was $70.42 \%$. Almost similar corresponding values were reported in another study done at Rajshahi region. ${ }^{22}$ In comparison to above-mentioned studies, the findings of microscopy in blood buffy coat of the present study was relatively higher. In this study, anti-k39 ICT were positive in $100 \%$ confirmed cases and over all it became positive in $94.3 \%$ of all febrile patients. Different other studies also found $100 \%$ positive results that well corresponds with findings of the current study. ${ }^{20,23}$ Manufacturers claimed up to $100 \%$ sensitivity with anti-k39 ICT strips, which value (100\% sensitivity) was also found in their pilot study. High rates of positive results with anti-k39 ICT could be explained as it is based on a protein antigen (K39) that appears as an immuno-dominant antigen in majority of KA patients giving rise to strong antibody response. ${ }^{24}$

No local study report to see LD bodies in blood buffy coat smears is available to compare with the findings of present study. Williams et al (1995) made comments that finding of 
the leishmania parasites in blood is sometimes possible in patients with Kala-azar from Kenya and India. ${ }^{25}$ The present study yielded LD body detection at a relatively higher rate $(>92 \%)$. This is well explained by the fact that density gradient centrifugation technique was adopted in this study using lymphoprep to prepare thick buffy coat in comparison to that found in Wintrobes tubes. As a result, more macrophages as well as more LD bodies became concentrated in buffy coat. So, the result found in this study might not be highly unexpected rather is encouraging. One limitation of the study is that confirmed negative afebrile controls could not be included because nobody in health agreed to sacrifice bone marrow or splenic aspirate for LD body detection. So, we could not calculate virtual sensitivity, specificity, positive and negative predictive values of LD body detection from blood buffy coat.

Analyzing the findings of the present study, use of modified blood buffy coat smear for confirmatory diagnosis of Kalaazar in endemic areas is recommended. This study also can inspire a prospective field for molecular and parasitic diagnosis of Kala-azar by an easy and accessible approach.

\section{References}

1. Haschiguchi Y. A global situation of Leishmaniasis. Research Report Series No. 6. Kochi Medical School, Namkoku, Japan; 2001: pp. 8-11.

2. Williams JE. Leishmania, Trypanosoma. In: Gillespie SH, Hawkey PM, editors. Medical Parasitology. A practical approach. UK: Oxford University Uress; 1995.

3. Chulay JD, Bryceson AD. Quantitation of amastigotes of Leishmania donovani in smears of spleenic aspirates from patients with Visceral Leishmaniasis. Am J Trop Med Hyg 1983; 32: 475-479.

4. Hockmeyer WT, Kager PA, Rees PH, Hendricks LD. The culture of Leishmania donovani in Schneider's insect medium: its value in the diagnosis and management of patients with Visceral Leishmaniasis. Trans R Soc Trop Med Hyg 1981; 75: 861-863.

5. Cummins AJ, Moody AH, Lalloo K, Chiodini PL. Development of a rapid latex agglutination test for the detection of Visceral Leishmaniasis. Trans R Soc Trop Med Hyg 1994; 88: 300.

6. Chowdhury MAJ. Clinical profile of Kala-azar in Rajshahi: A prospective study of 273 hospitalized patients during one year. JBCPS 1990; 8: 18-28.

7. Al-Masum MA, Evans DA, Minter DM, El-Harith A. Visceral leishmaniasis in Bangladesh: the value of DAT as a diagnostic tool. Trans R Soc Trop Med Hyg 1995; 89: 185-6.

8. Chandra J, Patwari AK. Diagnosis and treatment of Kala azar. Indian Pediatr 1994; 31: 741-8.

9. World Health Organization. Leishmania /HIV co-infection, south-western Europe, 1990-1998. WHO/LEISH/2000, 42. Geneva: WHO; 2000: pp. 1-12.

10. DGHS. Kala-azar: A training module for medical officers. Dhaka: DGHS; 1995: pp. 1-30.

11. Sundar S, Rai M. Laboratory Diagnosis of Visceral Leishmaniasis. Clin Diagn Lab Immunol 2002; 9: 951-958.

12. Cheesbrough M. Leishmanial parasite. In: Medical laboratory manual for tropical countries, volume I, 2nd ed. Cambridge: ELBS; 1987: pp. 272-288.

13. Evans D. Handbook on isolation, characterisation and cryopreservation of Leishmania. WHO special programme for research and training in tropical diseases, 1989: pp. 1-9.

14. Liarte DB, Mendonça IL, Luz FC, et al. QBC for the diagnosis of human and canine american Visceral Leishmaniasis: preliminary data. Rev Soc Bras Med Trop 2001; 34: 577-81.

15. World Health Organization. The Leishmaniases and leishmanial HIV co-infections. 1-4, Fact Sheet No. 116. Geneva: WHO; 1996.

16. Bryceson ADM. Leishmaniasis, In: Manson's tropical diseases, 20th edn. Philadelphia: WB Saunders Co. Ltd.; 1996: pp. 1213 $-1245$.

17. Ali A, Ashford RW. Visceral Leishmaniasis in Ethiopia. IV. Prevalence, incidence and relation of infection to disease in an endemic area. Ann Trop Med Parasitol 1994; 88: 289-93.

18. Benjamin B, Annobil SH, Bassuni WA. Diagnostic and management problems in childhood visceral leishmaniasis in south-western Saudi Arabia. Ann Trop Paediatr 1994; 14: 7-13.

19. Desjeux P. Human Leishmaniasis: Epidemiology and public health aspects. World Health Statistics Quarterly 1992; 45: 2-3.

20. Sarkar CB, Haqq MA. Kala-azar : as seen in a district general hospital of Bangladesh. Teachers Assoc J (Rajshahi) 1997; 10: 21-26.

21. Sundar S, Thakur BB, Tandon AK, et al. Clinicoepidemiological study of drug resistance in Indian Kala azar. Br Med J 1994; 308 (6924): 307.

22. Rahman MH, Khan AH, Rahman MM, Nazir FH. Clinical profile and therapeutic outcome of Kala-azar in hospitalized children in IPGMR. Bangladesh J Child Health 1995; 19: 117-120. 
23. Sundar S, Reed SG, Singh VP, Kumar PCK, Murray HW. Rapid accurate field diagnosis of Visceral Leishmaniasis. Lancet 1998; 351: 563-565.

24. Singh S, Gilman-Sachs A, Chang KP, Reed SG. Diagnostic and prognostic value of K39 recombinant antigen in Indian Leishmaniasis. J Parasitol 1995; 81: 1000-1003.
25. Williams DM, Carter KC, Baillie AJ. Visceral Leishmaniasis in the BALB/c mouse: a comparison of the in vivo activity of five non-ionic surfactant vesicle preparations of sodium stibogluconate. J Drug Target 1995; 3: 1-7. 